\title{
Performance of the Latest Generation Powerline Networking for Green Building Applications
}

\author{
Nirmalya Roy \\ Information Systems \\ University of Maryland Baltimore \\ County \\ nroyeumbc .edu
}

\author{
David Kleinschmidt \\ Bonneville Power Administration \\ U.S. Department of Energy (DOE) \\ dakleinschmidtabpa.gov \\ Behrooz Shirazi \\ Electrical Engineering and \\ Computer Science \\ Washington State University \\ shiraziewsu.edu
}

\author{
Joseph Taylor \\ Electrical Engineering \& \\ Computer Science \\ Washington State University \\ j.taylor@wsu.edu
}

\begin{abstract}
Green building applications need to efficiently communicate fine-grained power consumption patterns of a wide variety of consumer-grade appliances for an effective adaptation and percolation of demand response models in the home environment. A key hurdle to the widespread adoption of such demand response policies in these appliances is the lack of efficient connectivity to a local area network. One solution is delivering telemetry data over existing electrical infrastructure to which the devices are already connected. The use of existing wiring produces a simple and cost-effective solution, avoiding many issues observed with wireless mesh networks (such as islands and bottlenecks), while helping to vacate increasingly congested spectrum. In this paper we explore the feasibility and efficacy of Powerline Communications (PLC) as a backbone of wireless communications in a home environment. We evaluate the behavior of several state-of-the art PLC modems using end-to-end measurements to establish their performance and throughput characteristics. Our preliminary results suggest that PLC is a promising technology for low-bandwidth hungry green building applications but more in depth study is required before making large-scale smart grid deployment.
\end{abstract}

\section{Categories and Subject Descriptors}

B.4.1 [Hardware]: Input/output and Data Communications-Data Communications Devices; C.2.5 [Computer Systems Organization]: Computer Communication Networks-Local and Wide-Area Networks

(c) 2013 Association for Computing Machinery. ACM acknowledges that this contribution was authored or co-authored by an employee, contractor or affiliate of the United States government. As such, the United States Government retains a nonexclusive, royalty-free right to publish or reproduce this article, or to allow others to do so, for Government purposes only.

BuildSys'13, November 14 - 15 2013, Roma, Italy

Copyright (C) 2013 ACM 978-1-4503-2431-1/13/11 ...\$15.00

\section{General Terms}

Measurement, Performance, Experimentation, Reliability, Standardization

\section{Keywords}

Powerline Communications, PLC, Smart Grid, Green Building

\section{Introduction}

Smart sensors and plugs are being actively installed to connect a growing number of consumer-grade appliances to smart grid applications. The goal, in most cases, is to get better control over the fine-grained usage of those appliances and integrate their demands more seamlessly within the power grid. Most of these sensors and smart plugs connect wirelessly for data collection, communications, and control over the smart grid with a minimal bandwidth overhead [12][14][20]. An alternative solution (available since 2001) is Powerline communication (PLC), which superimposes a high frequency carrier signal onto the home electrical wiring to provide a wired connection medium. Adaptors then convert the signal at wall outlets to Ethernet for use with appliances [9]. The promise of this technology is the avoidance of wireless spectrum crowding, greater bandwidth and reliability compared to wireless networks, increased security, and the usage of the existing electrical infrastructure which simplifies the deployment of smart grid technologies. However, previous research has shown that this technology does not live up to manufacturers' performance ratings, and other electrical devices (primarily motors) on the home power network significantly affect performance [15]. Given that a large percentage of consumer grade appliances are motor loads, it seems the appliances themselves could inhibit the viability of the electrical system as a communication path to the smart grid applications. Another complaint was that these devices used proprietary protocols not published by vendors. As the technology has developed further, more protocol information has become public in IEEE standard 1901, and the theoretical data rates have increased [11]. Vendors have also designed filters to reduce the impact of electrical noise on the performance of PLC networks. In this paper, 
the latest generation of adaptors are tested to see if data rates have increased significantly, and if the filter technology can improve the lackluster performance of previous generation models, particularly in the presence of motor based appliances. Since wireless technology has also continued to improve, the latest PLC adaptors are compared to more modern wireless technology. The main focus of conducting the performance tests is to determine the suitability of these devices for deep demand response management in smart grid applications.

In this paper, we outline an overview of PLC networking devices to help guide the architectural choices for future PLC network design. We provide detailed experimental results based on some of the newly designed state-of-theart PLC hardwares for untethering the capabilities a highbandwidth Ethernet PLC [10] and a low-bandwidth Cypress PLC modem [4] could provide. Compared to the previous work [15][17], we present a thorough investigation on the effects of highly-detrimental noise over powerline communication in a normal home. This study is useful in future for PLC deployment and decision making in case of measuring power consumption data from a variety of plug load outlets in a home environment. We have investigated such sources of noise and looked at how they might impact reliability and throughput in the very worst case conditions. Given a variety of smart grid and green building applications in the horizon with different reliability and bandwidth requirements, our results attest to the baseline of different design choices for future researchers in this area as powerline communications evolve for smart grid control and communications.

The rest of the paper is organized as follows. Section 2 discusses the background and standardization effort of powerline communications. Section 3 describes the development in powerline communications for high-bandwidth intensive applications, equipment and testing methodologies with experimental setup and results. Section 4 describes our experiments for low-bandwidth intensive applications including the noise, range, connectivity and distance testing for different appliances. Section 5 summarizes our findings and Section 6 identifies future research directions and concludes our work.

\section{PLC Background and Standardization}

Powerline communications is an evolving technology that is corroborating yet another renaissance [2][19]. There exist several competing and cooperating standards that describe both parts of power line networks and complete interoperable systems [13]. A few standards observed to be in common use for home network devices are described below.

IEEE 1901 [11] defines 2 MAC standards and 2 physical interface standards. The different MAC standards are reportedly extant to suit the unique needs of home networking verses WAN access, with each of the aforementioned being the basis for their respective MAC. The two physical interfaces are the "FFT" PHY and the "Wavelet" PHY. The former is used in the "HomePlug" standards, as described next.

HomePlug [9] is an Oregon-based producer of licensable standards. Along with development of the HomePlug standards, they also perform IEEE 1901 compliance test- ing. HomePlug implements an IEEE 1901-compliant complete networking solution, using the IEEE 1901 FFT PHY. Some end user niceties are also included in compliant devices; password-derived encryption and QoS are part of the standard. Modulation is done with FFT OFDM, using channel modulation schemes that adapt to line conditions automatically. Turbo coding forward error correction is used to reduce the incidence of required retransmits. The first HomePlug standard ("HomePlug 1.0,") has since antiquated for a non-backwards compatible standard "HomePlug AV." HomePlug AV [9] is the HomePlug standard currently in common use. A new standard "HomePlug AV2" [10] has been announced, with compliant devices expected in the second half of 2013.

G.hn [8] is a standard developed by the International Telecommunications Union's standardization sector. Modulation schemes are similar to the methods used by HomePlug-compliant devices, but the two standards are not interoperable. G.hn uses FFT OFDM, with QAM-modulated channels. The number of points in the QAM constellation are adjusted for line conditions, reaching a peak of 4096QAM in ideal conditions. Like HomePlug, G.hn also offers security. G.hn can operate over both AC and DC power lines, and it can provide the communication infrastructure required for smart grid applications in residential, commercial and industrial environments.

OFDM is part of all the aforementioned standards, and its usefulness is easy to understand when the challenges of EMI noise on power lines is considered. The use of multiple, independent channels provides inherent noise tolerance, as even higher-noise devices will disrupt some but likely not all of the channels in use. Both HomePlug and G.hn use forward error correction for obvious reasons. Automatic adjustment for the severity of line noise is another common theme, seen in both the HomePlug and G.hn standards.

\section{Developments in Powerline Communica- tions for High-Bandwidth Applications}

Beginning with the first Homeplug 1.0 standard in 2001, the initial data rates for PLC were roughly 14-85 Mbps. This was comparable to wireless speeds offered at the time, but not suitable for modern use. In 2005, the Homeplug AV standard debuted, which promised PHY layer data rates of 200 Mbps and MAC layer data rates of 80 Mbps operating in a frequency band from 2-28 MHz [15]. More recently, adaptors capable of PHY layer data rates of $500 \mathrm{Mbps}$ have been developed and are the subject of this paper. These adapters use proprietary methods that widen the frequency band to increase throughput (2-80 MHz) [10]. Detailed documentation of these products is also difficult to find, as in prior research [15].

With the increasing focus on energy conservation, numerous groups have developed interest in PLC technology to facilitate smart appliance and HVAC monitoring and control. The benefit would be the optimization of energy consumption at the household level and developing a comprehensive smart grid system reaching into every plug load outlet in a home or building so that all devices can participate in energy conserving strategies. This has pushed the development of 


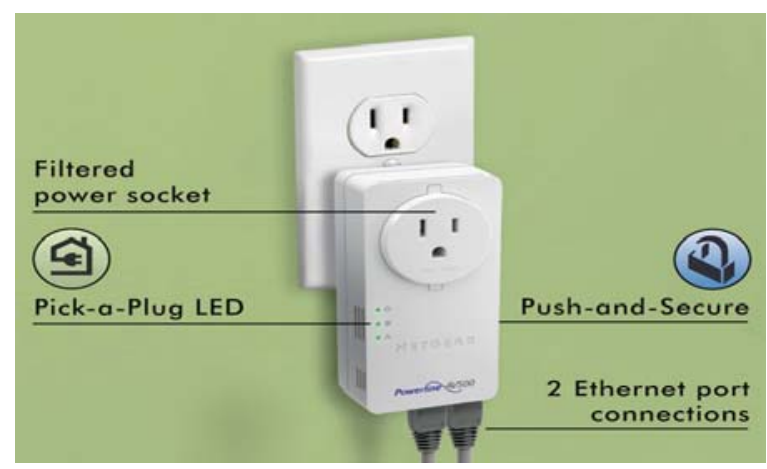

Figure 1. Netgear Powerline 500 Mbps Nano Kit with Pass Thru and Dual Ports

IEEE standard 1901, which helps define the communication protocol. Detailed specifications of the PHY and MAC layers are provided in [11]. Of interest here is the frequency range of operation $(2-80 \mathrm{MHz})$, well above the power frequency of $60 \mathrm{~Hz}$. However, other electronic devices in the home generated high frequency harmonics that can cause serious issues for the operation of PLC devices. To counter this, device makers have developed filters to reduce the noise injected into the line by various household appliances.

As the Homeplug AV standard has developed over the years, so too has wireless networking technology. The common wireless standard of today is $802.11 \mathrm{n}$, and is built into many consumer devices. While previous generation $802.11 \mathrm{~g}$ operated strictly in the $2.4 \mathrm{GHz}$ band, $802.11 \mathrm{n}$ can operate in the $2.4 \mathrm{GHz}$ or $5 \mathrm{GHz}$ bands. The $5 \mathrm{GHz}$ band promises reduced interference due to less competition, but the signal has greater difficulty traveling through solid objects. Furthermore, both frequency bands support up to 3 simultaneous data streams for increased bandwidth, offering a theoretical data rate of $450 \mathrm{Mbps}$ [3]. This specification compares well to the latest PLC devices. However, it is not guaranteed that all devices will support this highest level of speed just as with PLC devices. Finally, the bandwidth requirements of some of the applications are much higher. For example, 1080P Netflix streaming requires up to 8 Mbps throughput [16], while streaming uncompressed HD video can require up to 22 Mbps [1]. This is the requirement for a single device, which doesn't include the bandwidth used by any other devices on the network for smart grid control and communications.

\subsection{Equipment and Testing Methodology}

Numerous vendors supply PLC hardware in the consumer market, with various speed ratings offered. The model chosen for this study was the Netgear XAVB5602 which conforms to HomePlug AV, a dominant standard in powerline communication as shown in Figure 1. This adaptor provides a PHY layer rating of $500 \mathrm{Mbps}$, and also includes a filtered outlet on each adaptor. The adaptor has 2 Ethernet ports in order to connect multiple devices without a hub or switch. Independent filters were originally desired, but finding them proved to be a challenge (both in store and online). Specialty ordering from the manufacturer was required which was outside the scope of this work.
Two adaptors were purchased to evaluate the performance of the devices. While testing of more adaptors might be beneficial, it is unlikely that a home user would implement a complete PLC network. Again, this is due to the convenience of wireless networking and the ubiquitous nature of wireless adaptors. Most likely, PLC devices will be employed to reach a section of the home not covered well by the wireless signal or to wire up the appliances more relevant to smart grid control and communications or to provide a more reliable connection for high definition video streaming at a single device.

The testing environment was a 1300 sq. ft., 2 story townhome built in 1995 . The townhome simulates a very realistic home testing environment, with all common appliances such as refrigerators, microwave ovens, televisions etc. Being a townhome, it also suffers from frequency crowding of nearby wireless networks. This has resulted in video streaming problems for current residents. A $50 \mathrm{ft}$. extension cord was also used to somewhat isolate the adaptors from household electrical noise during some of the tests. Finally, laptops equipped with dual stream $802.11 \mathrm{n}$ adaptors (300 Mbps) were employed for testing of the adaptors and wireless comparison purposes.

\subsection{Experimental Setup and Results}

In order to quantify the performance characteristics of the latest generation of powerline networking adaptors for highbandwidth seeking applications, numerous tests needed to be conducted. Specifically throughput and round trip time (RTT) are the most important metrics to consider, since they indicate the network's capability of transferring large quantities of data with limited latency. Ping tests and iperf software were used to capture these metrics during a distance test and various transfers set up on the extension cord with household appliances operating.

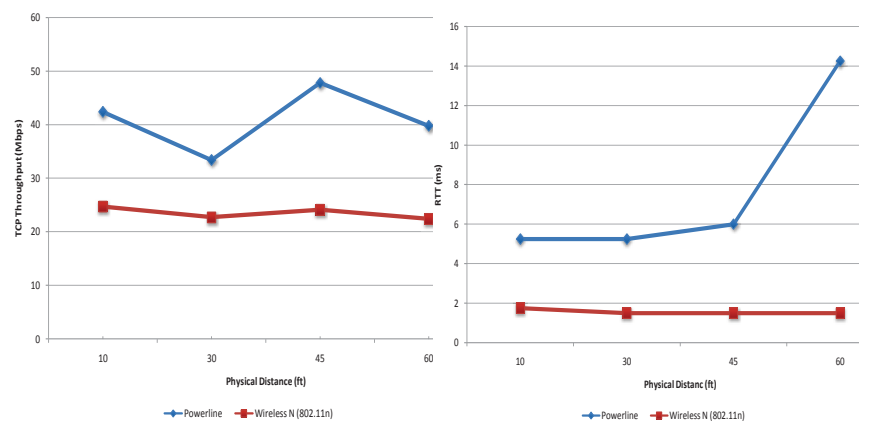

Figure 2. TCP throughput Figure 3. RTT as a function as a function of distance of distance

\subsubsection{Distance Test}

In this test the adaptors were set up at various outlets within the townhome at increasing distances. These are direct distances between outlets, not electrical cabling distances. Both single floor and multiple floor tests were carried out to demonstrate a wide range of applications. Throughput and RTT were captured at the various outlet positions. At those same positions, an ad hoc wireless network was set up between the two laptops to test the wireless connection for comparison purposes. 
As shown in Figure 2, the PLC adaptors provide higher throughput than the wireless connection, but the connection seemed less consistent. It was also nowhere near the rated throughput. Figure 3 reinforces the consistency of the wireless connection with respect to RTT, and interestingly the RTT time increases significantly with distance for the PLC adaptors. This runs contrary to the results found in [15].

\subsubsection{Powerline Noise From Appliances}

As has been noted multiple times by previous research efforts and the manufacturers themselves, noise in the powerline network can significantly deteriorate the performance of PLC devices. To quantify this, three common household appliances were picked to test their respective effects on performance: a coffee grinder, a large bakery mixer, and a vacuum cleaner. The wattages of the three devices are shown in Table 1.

Table 1. Wattage of appliances used for testing

\begin{tabular}{|c|c|}
\hline Device & Wattage \\
\hline Coffee Grinder & 175 \\
\hline Bakery Mixer & 425 \\
\hline Vacuum & 1400 \\
\hline
\end{tabular}

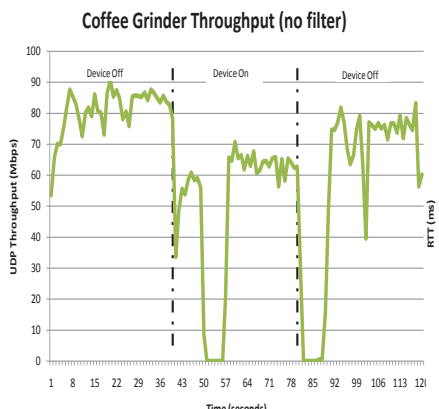

Figure 4. Coffee grinder UDP throughput without filter

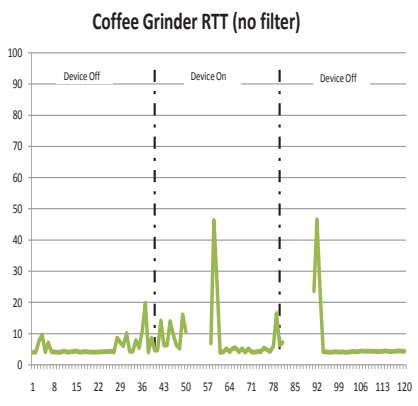

Figure 5. Coffee grinder RTT test without filter
To investigate the impact of these appliances, the two adaptors were connected at either end of the extension cord with the appliance connected to the same wall outlet as the extension cord (a splitter was used). A saturating UDP stream was set up between the two nodes and throughput data was recorded with the appliance off for the first $40 \mathrm{sec}-$ onds. Then the appliance was turned on for 40 seconds, followed by another 40 seconds turned off. This portion was conducted using iperf. Then, the same test was performed with a series of pings ( $64 \mathrm{~kb}$ packets, $128 \mathrm{~ms}$ TTL) to record the impact on RTT of the appliances. Finally, these tests were rerun with the appliance plugged into the filtered outlet of the node at the wall.

Figures 4-5 show the UDP throughput and RTT results for the coffee grinder plugged into the regular wall socket. The PLC adaptors have serious performance problems when the coffee grinder is running, with starting and stopping of the motor causing throughput to drop all the way to zero before recovering. This signifies the adaptors' response to the noise by changing its modulation scheme. RTT is also significantly affected, with packets being lost due to timeout after both starting and stopping of the grinder.
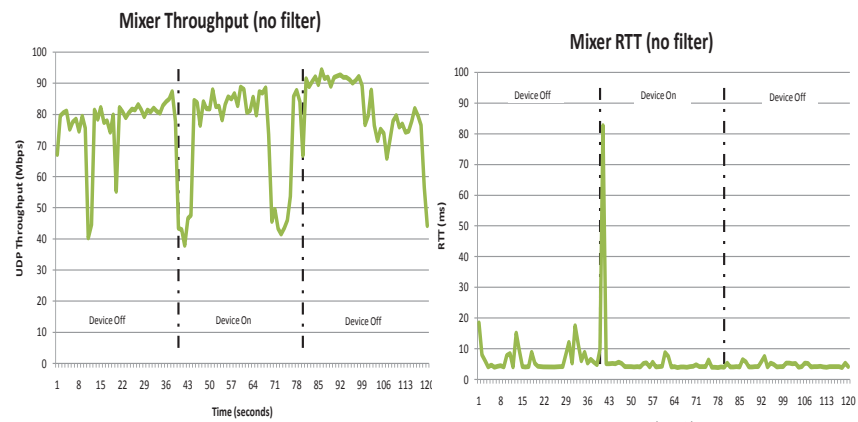

Figure 6. Bakery mixer UDP throughput test without filter

Figure 7. Bakery mixer RTT test without filter

Figures 6-7 show the UDP throughput and RTT results for the bakery mixer when plugged into the regular wall socket. While the wattage of the motor is rated higher than the coffee grinder, the impact of its operation is much less significant on network performance. Throughput and RTT are still affected by motor starts and stops, but the connection is maintained with no packets lost to timeout. Since it is a larger, more slowly operating motor, it is likely that it does not emit as much high frequency noise on the line as the small, fast spinning coffee grinder. Also, nothing was actually being mixed in the mixing bowl, providing no mechanical load for the motor. A mechanical load may change the noise profile generated by the mixer.

Results for the vacuum plugged into the regular wall socket are not shown due to the fact that turning on the vacuum completely shut down the connection, requiring a hard reset for the connection to be established again. This exhibits the worst-case scenario for powerline noise, with high RPM, high power motors completely interrupting communication with no automatic recovery.
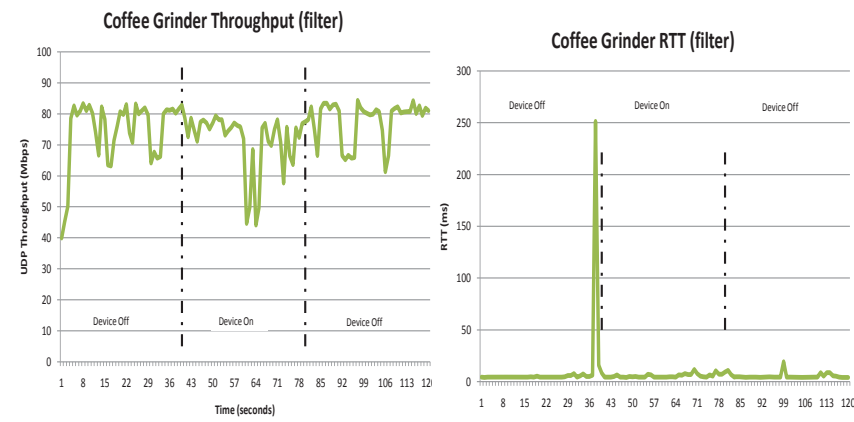

Figure 8. Coffee grinder UDP throughput with noise filter

Figure 9. Coffee grinder RTT test with noise filter

Figures 8-9 show the UDP throughput and RTT for the coffee grinder when plugged into the filtered outlet of the adaptor. The filter has a very positive effect on performance, with throughput maintained at a much higher level $(\approx 30 \%$ improvement on average during the device 'On' state) and no 
packet loss due to timeout during the RTT test. It does not appear that the noise is completely eliminated, but significant performance gains are measured. Figures 10-11 show the same results for the bakery mixer, with the motor start and stop having seemingly no impact on throughput and RTT when the mixer is plugged into the filtered outlet.

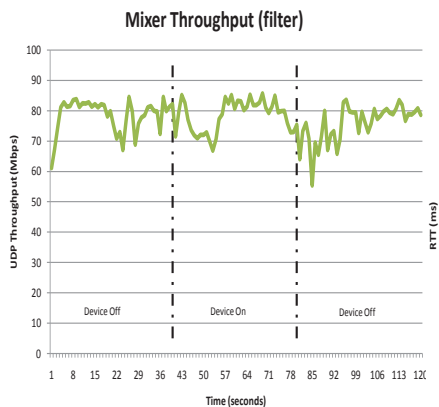

Figure 10. Bakery mixer UDP throughput test with noise filter

Finally, Figures 12-13 show the UDP throughput and RTT results of the vacuum plugged into the filtered outlet of the adaptor. Once the motor is turned on the adaptor attempts to compensate but the connection completely drops the rest of the time for the throughput test. It is unclear why the connection was unable to recover during the 40 seconds following motor shutoff, but a hard reset was not required after an additional minute of waiting. The ping test was able to run throughout the operation of the motor, with quite a bit of variation in RTTs recorded.
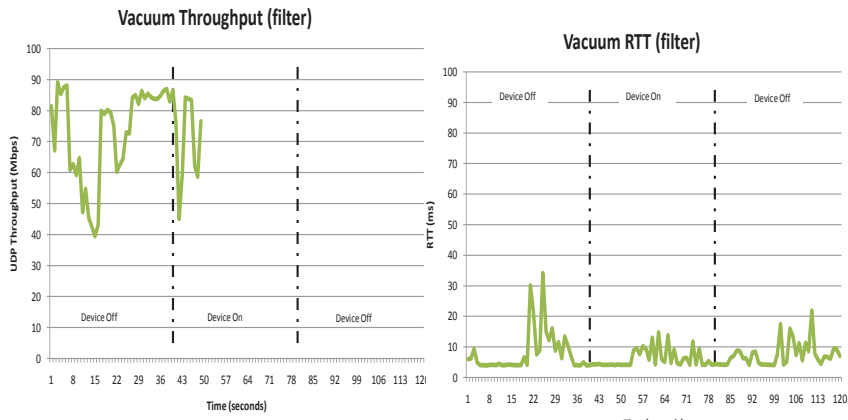

Figure 12. Vacuum UDP throughput test with noise Figure 13. Vacuum RTT filter

\subsubsection{Multiple Simultaneous Connections}

Since the adaptors contained two Ethernet ports each, it was of interest to test the throughput of multiple TCP connections between two adaptors. This was achieved by connecting two laptops running iperf to each adaptor (total of 4 laptops). Iperf throughput tests are set up with one laptop acting as the client, and the other as the server. Two tests were run, with the first being both laptops at one end acting as servers and the other end acting as broadcasting clients. The TCP streams would then be traveling in the same direction. Then, both a client and a server were placed at each end,
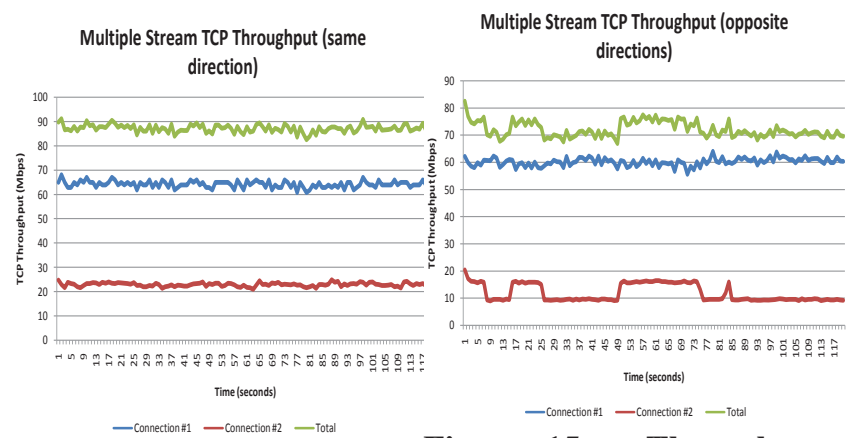

Figure 14. Throughput of Figure 15. Throughput two TCP connections origi- of two TCP connections nating in the same node originating in the opposite nodes

with the TCP streams traveling opposite directions. This test was set up to analyze the adaptors ability to both send and receive packets for multiple connections simultaneously.

Figure 14 shows the TCP throughput of each connection with the streams going in the same direction, as well as the total throughput. Interestingly, the throughput is not split evenly between the two connections, and does not exceed $100 \mathrm{Mbps}$. This calls into question the increased speed ratings of these new adaptors. Figure 15 shows the TCP throughput of each connection when running in opposite directions, along with the total throughput. Again, the throughput is not evenly divided, and it seems that throughput suffers (especially for connection no. 2) for streams going in opposite directions. This could be a result of packet collisions. It should be noted that connection no. 1 originates from the end of the extension cable farthest from the wall outlet in both tests. Connection no. 2, however, originates at the wall socket for the second test. This could also account for the reduced performance of connection no. 2 in the second test since electrical noise would be more prevalent at the wall socket.

\section{Developments in Powerline Communica- tions for Low-Bandwidth Applications}

While home networks require a great deal of bandwidth as explained in previous sections, many other applications are much less demanding. Examples include in-home power consumption telemetry or room-to-room signaling between appliances. In such applications a lower baud rate connection with a more robust resistance to noise may be preferable. In exploring this, we tested with the Cypress Semiconductor CY8CPLC10 modem [4]. Avoiding the much more complex OFDM implementations of powerline Ethernet devices, the CY8CPLC10 uses FSK modulation at $133.3 \mathrm{kHz}$ and 131.8 $\mathrm{kHz}$ for logic 0 and 1 respectively [7].

The Cypress PLC solution enables secure and reliable communication over powerline. It integrates powerline PHY modem with optimized filters and amplifiers to operate with high voltage and low voltage powerlines. It also supports bidirectional communication with optional acknowledgement based signaling. In case of data packet loss due to louder noise on the powerline, the transmitter has the capability to retransmit the data. The powerline network protocol 
Table 2. A snapshot of the collected noise (decibel microvolts) traces using PLC. Baseline - operating computer, necessary periphery, Lamp - 40 watt CFL, Vacuum Cleaner - "ShopVac"

\begin{tabular}{|l|l|l|l|l|l|l|l|l|l|}
\hline $\begin{array}{l}\text { Noise } \\
(\mathrm{dB} \mu \mathrm{v})\end{array}$ & Baseline & Lamp & Projector & $\begin{array}{l}\text { Air } \\
\text { Conditioner }\end{array}$ & $\begin{array}{l}\text { Stovetop } \\
\text { Fan }\end{array}$ & $\begin{array}{l}\text { Micro } \\
\text { wave }\end{array}$ & $\begin{array}{l}\text { Robotic } \\
\text { Cutter }\end{array}$ & $\begin{array}{l}\text { Vacuum } \\
\text { Cleaner }\end{array}$ & $\begin{array}{l}\text { Stovetop Fan }+ \\
\text { Air Conditioner }\end{array}$ \\
\hline \hline Average & 87 & 87 & 87 & 86 & 86 & 86 & 86 & 86 & 86 \\
\hline Highest & 99 & 93 & 90 & 90 & 87 & 87 & 87 & 99 & 87 \\
\hline Lowest & 87 & 87 & 87 & 80 & 80 & 80 & 80 & 80 & 80 \\
\hline
\end{tabular}

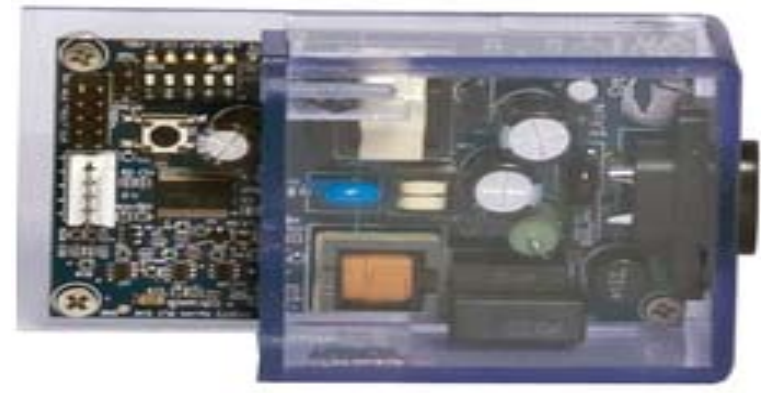

Figure 16. Cypress CY3272 evaluation board
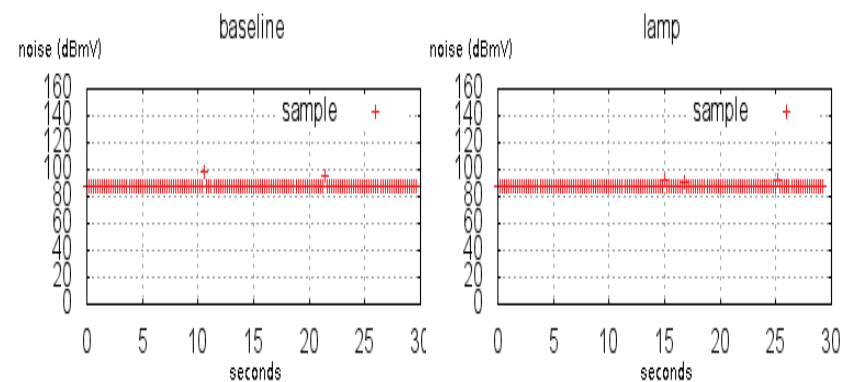

Figure 17. minimal noise Figure 18. A 40 watt lamp baseline noise in the line

also supports 8-bit CRC for error detection. A Carrier Sense Multiple Access (CSMA) scheme is built into the data link layer. It avoids collision between packet transmissions from different nodes on the powerline, supports multiple masters, and enables reliable communication for large-scale deployment [18].

\subsection{Equipment and Testing Methodology}

The Cypress PLC modem exposes an I2C interface for sending data and controlling the transceiver. Given that board production would be particularly time consuming, we elected to instead use a Cypress evaluation device-the CY3272 as shown in Figure 16. This board holds the modem IC along with a power line coupling interface. Reception was done using a Cypress Semiconductor CY3274 evaluation board. This board holds a CY8CPLC20 (PSoC microcontroller with a CY8CPLC10 on die) along with the power line coupling interface and serial interface for communicating with the PC. Software used was adapted from Cypress example code [6]. The transmitting and receiving node address each other by hard coded node identifiers.
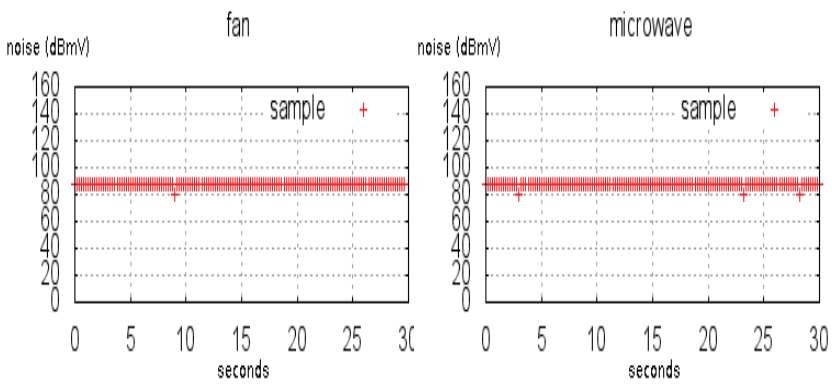

Figure 19. A range hood Figure 20. A 700 watt mifan noise in the line

crowave noise in the line

\subsection{Experimental Setup and Results}

The test circuit was a Cypress CY3272 evaluation kit, which includes the CY8CPLC10 and power line coupler, and pins that expose the CY8CPLC10 I2C interface. Given the limited bandwidth, the overhead of IP was prohibitive in testing. Instead we tested using transmission raw bytes between a pair of modems. In testing range it was found that reception was $100 \%$ reliable regardless of where they were attached in the home. Noise testing was done with a variety of devices as shown in Table 2, and then left to run across a 24-hour period as shown in Figure 23.

\subsubsection{Range Testing}

We tested our prototype both in the WSU smart environment lab and in the town-home, and found performance to be reasonable in both cases. While information about actual line distances and wiring was unavailable, we had success communicating in all parts of the apartment, all parts of the lab, and observed successful transmission of packets even (some distance down the hallway.)

\subsubsection{Noise Testing}

The Cypress IC allows for noise testing in the frequencies used by the transceiver using Cypress's PLC Control Panel software [5]. In the apartment where the previous range testing was done we tested for impedance of communication and for the noise measured with a small set of household devices in use. Our results showed $100 \%$ reliability in reception in all cases, and very little noise on the relevant frequencies. We sampled noise at 200 millisecond intervals, and recorded noise measurements as decibel millivolts. Figure 17 shows the baseline noise over time where only the noise monitoring equipment is operating. Figure 18, 19, 20, 21, 22 represent noise over time respectively for a lamp, fan, microwave, air conditioner, and fan and air conditioner together.

Noise held at 87 decibel millivolts for nearly every sample, with rare deviations. These results seem to suggest very 

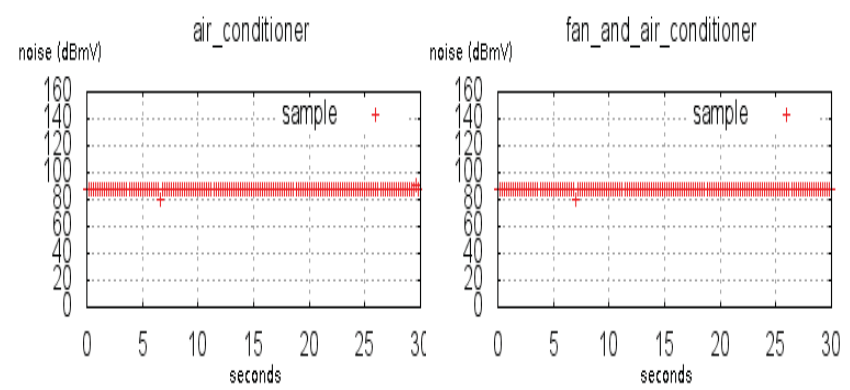

Figure 21. A large portable Figure 22. A range hood air conditioner noise in the fan and air conditioner line noise in the line

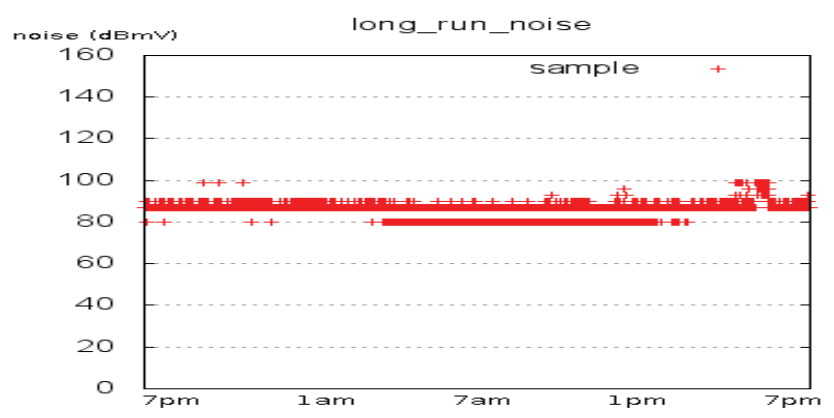

Figure 23. Ambient line noise across 24 hours

strong noise immunity for the PLC modem. Next we observed background noise across a 24-hour period, again at the same apartment. Samples were made in 2 - second intervals from 7 PM on July 28, 2013 to 7 PM on July 29, 2013.

There was no apparent correlation between appliances in use at the apartment and the noise measured patterns observed are suspected to be external of the residence. The graph starts at 7 PM and ends at 7 PM 24 hours later. The area of low noise was from approximately $5 \mathrm{AM}$ to $5 \mathrm{PM}$ without any apparent cause.

\subsection{Logical Impact}

Our main focus of conducting these various performance tests is to determine the suitability of PLC devices for deep demand response management for smart grid applications for both most taxing and non-taxing type of data transfer applications. In this work we intend to find out the reliable transmissions on the low-bandwidth PLCs in conditions where high-bandwidth transmissions are hindered or of a concerning reliability, and we are able to confirm that it appears largely invulnerable to the common household noise issues (vacuum, fan, etc.) that may have been concerning to the developers looking at PLCs as a potential communications method. Numerous reliability, noise and throughput tests have been performed in WSU smart homes to examine usability of power line networking devices and what considerations and conditions would drive a designer to choose a low or high bandwidth connection. Apparently while transferring data in bulk we observe that PLC Ethernet is an obvious choice with compromised reliability in presence of noise for certain cases, but for data with a lower transferring rate
Cypress PLC is a more viable choice. Furthermore, there may be situation where needs are somewhere in between, and therefore the data we collected might help drive decisions if a hardware developer is otherwise uncertain.

\section{Discussion}

Compared to previous studies, it does not appear that throughput or RTT performance has increased at all with the latest generation of adaptors for high-bandwidth prone application. If anything, more variation in performance was observed in this study than seen previously. Compared with the latest generation of widely adopted Wifi, PLC adaptors still promise greater performance in low noise environments, but at the risk of performance issues when more noise is present. Since throughput rates above $20 \mathrm{Mbps}$ were observed using $802.11 \mathrm{n}$, this seems sufficient for many applications.

On the other hand, the performance boost of the adaptors when the appliances were plugged into filtered outlets is promising. In the event that a wireless network did not provide adequate performance, independent adaptors could be ordered for the major appliances in the home to filter out the noise that they generate. This study tested the worst case scenario of the appliances plugged into outlets very close the PLC adaptors, which may not be the case in an actual home. It should be noted, however, that the operation of the dishwasher (located $15 \mathrm{ft}$. away from the testing outlet) caused the PLC network to completely disconnect. Only when the dishwasher was shut off could the adaptors reestablish a connection with each other. This just reinforces the susceptibility of the adaptors to line noise. It should also be mentioned that after testing, it was discovered that both Ethernet ports on the adaptors are only rated for $100 \mathrm{Mbps}$. Instead of Gigabit Ethernet ports to take advantage of the rated $500 \mathrm{Mbps}$ throughput, it seems the manufacturer built in a bottleneck for network performance. Therefore, it would be impossible to see throughput above the rating of the Ethernet connection for a single connection. However, it was observed that even the sum of multiple connections could not provide TCP throughput in excess of $100 \mathrm{Mbps}$, calling into question the device ratings. Online discussions have noted that the 500 Mbps rating is actually a "whole home" rating for all connected adaptors, but this is not stated in the product literature. This makes the rating seem deceptive for the average home user who will likely be disappointed in the performance.

Previous work has explored the viability of PLC technology mostly for high-bandwidth sensitive applications [15], however we are unaware of any investigation comparing and evaluating the plausibility of PLC networks for lowbandwidth prone applications such as power consumption telemetry over the existing mains wiring in a building environment. Our low-bandwidth sensitive results seem to suggest that communicating power consumption telemetry via powerline networking is a promising solution. The high noise immunity and tested range would work well for inbuilding consumption telemetry.

\section{Conclusions and Future Work}

With the increasing focus on energy conservation, numerous groups have developed interest in PLC technology to facilitate smart appliance and HVAC monitoring and control. 
The benefit on integrating the PLC technology would be the optimization of energy consumption at the household level and developing a comprehensive smart grid system reaching into every plug load outlet in a home or building so that all devices can participate in energy conserving strategies. In this study, we hypothesize that powerline Ethernet would provide rapid but not perfectly reliable transmission of data in presence of noise, and that has been confirmed with our experiments. We also hypothesize that the Cypress PLC (based on the way it transfers data, low-bandwidth FSK) would provide a much better noise immunity which indeed has been the case. Through this study we conclude that the Cypress modem is largely immune to the normal home noise issues that were observed in the Ethernet modules. In general PLC adaptors promise greater performance in low noise environments, but compromise the performance when more noise arise.

Additional testing is needed to further analyze the performance of HomePlug devices in a greater set of circumstances. Given the difficulty finding independent filters at retailers, they could be ordered and a more thorough analysis of their effectiveness could be completed. Testing of additional adaptors (beyond two) could be beneficial, but as mentioned previously this is an unlikely scenario for home use. A further search found that other vendors provide adaptors with Gigabit Ethernet ports, which could be tested to see if the Ethernet port is the bottleneck or if the PLC technology is limiting throughput.

The Homeplug Alliance has also announced the next generation of the technology, known as Homeplug AV2 [10]. This provides an alliance standard of 500 Mbps throughput, instead of the proprietary schemes individual manufacturers have employed to rate their devices at this level. These devices are expected to become available in the latter half of 2013. Retesting of these new devices under similar conditions as this study could be beneficial. In the same study, the next generation of Wifi (802.11ac) could also be tested. These new Wifi devices are already available, but mass adoption has yet to occur. The 802.11 ac technology is rated for a maximum throughput of $1300 \mathrm{Mbps}$, much higher than the Homeplug AV2 standard. However, these ratings are achieved in the $5 \mathrm{GHz}$ band, which has known issues with range.

Given the lack of performance improvements, the difficulty in obtaining appliance filters, and the relatively high cost of PLC adaptors (the testing pair cost 110), this technology still does not seem ready for wide adoption by high-bandwidth intensive applications whereas it holds great promise for low-bandwidth intensive applications. However, we believe that a larger scale deployment is necessary to validate the PLC modems' noise, range and packet drops resiliency in a more realistic setting. Our initial results are promising in considering the PLC as a viable low-level protocol layer solution for fine-grained power monitoring of the appliances in a green building environment, but adding the concurrent power metering over the data communications may complicate this already tricky design space. We plan to investigate both the metering and communication of the power telemetry as a next step besides performing more large scale performance evaluations.

\section{Acknowledgements}

We thank the anonymous reviewers for their constructive feedback and our paper shepherd Steven Lanzisera for his detailed comments to improve the quality of the paper. This work is partially supported by the National Science Foundation Award \#1255965 ("CSR-EAGER").

\section{References}

[1] Blu-Ray Disc Association. Blu-Ray White Paper, http://www.bluraydisc.com/en/technical/technicalwhitepapers/general.aspx

[2] D. Clark. "Powerline communications: finally ready for prime time?", IEEE Internet Computing, 2(1), 1998.

[3] Cisco Systems, Inc. 802.11ac White Paper, www.cisco.com/en/US/prod/.../white_paper_c11-713103.html

[4] CY8CPLC10: Powerline Communication Solution. http://www.cypress.com/?rID=38236

[5] Cypress Powerline Communication Control Panel GUI. Cypress Semiconductor. http://www.cypress.com/?rID=38135

[6] AN54416: Using CY8CPLC20 in Powerline Communication (PLC) Applications. Cypress Semiconductor http://www.cypress.com/?rID=37951.

[7] CY8CPLC10 datasheet. http://www.cypress.com/?docID=26408

[8] HomeGrid Forum, http://www.homegridforum.org/

[9] HomePlug Alliance. HomePlug AV White Paper, http://www.homeplug.org/.

[10] HomePlug Alliance. HomePlug AV2 White Paper, http://www.homeplug.org/.

[11] IEEE. Standard 1901-2010. http://standards.ieee.org/findstds/standard/19012010.html

[12] X. Jiang, M. Van Ly, J. Taneja, P. Dutta, and D. Culler, "Experiences with a high-fidelity wireless building energy auditing network", Proceedings of the 7th ACM Conference on Embedded Networked Sensor Systems, ACM SenSys'09. NY, USA, pp. 113-126.

[13] M. K. Lee, R. E. Newman, H. A. Latchman, S. Katar, and L. Yonge. "Homeplug 1.0 powerline communication LANs protocol description and performance results", International Journal of Communication Systems, 16(5):447473, 2003.

[14] J. Lifton, M. Feldmeier, Y. Ono, C. Lewis, and J. A. Paradiso. "A platform for ubiquitous sensor deployment in occupational and domestic environments", 2007.

[15] R. Murty, J. Padhye, R. Chandra, A. R. Chowdhury, and M. Welsh, "Characterizing the End-to-End Performance of Indoor Powerline Networks", Harvard University Technical Report, 2008.

[16] Netflix, Inc. "Internet Connection Speed Requirements", https://support.netflix.com/en/node/306

[17] P. Pannuto and P. Dutta, "Exploring powerline networking for the smart building", IP+SN workshop, 2011.

[18] R. Parsani, "Cypress Powerline Communication Performance Analysis", Cypress Semiconductor White Paper.

[19] N. Pavlidou, A. Han Vinck, J. Yazdani, and B. Honary. "Power line communications: state of the art and future trends", IEEE Communications Magazine, 41(4): 34-40, Apr. 2003.

[20] B. Priyantha, A. Kansal, M. Goraczko, and F. Zhao. "Tiny web services: Design and implementation on interoperable and evolvable sensor networks", Proceedings of the 6th ACM Conference on Embedded Networked Sensor Systems, ACM SenSys 2008. 